\title{
Fast-Acting Insulin Aspart: The Rationale for a New Mealtime Insulin
}

\author{
Marc Evans · Mathew Wilkinson · Angeliki Giannpolou
}

Received: July 23, 2019 / Published online: September 4, 2019

(C) The Author(s) 2019

\begin{abstract}
Attenuating postprandial hyperglycaemia is a critical factor in the achievement of optimal glucose control. Prandial insulin analogues have been developed to replicate the physiology of normal endogenous insulin secretion and action, with the aim of limiting postprandial glucose excursions. There is still, however, a significant unmet need, with many people failing to achieve desired glycaemic control targets despite the current armamentarium of prandial insulin analogues. Such insulins have a delayed onset and a longer duration of action than endogenous insulin production. There has been considerable focus on attempts to accelerate the time-action profile of prandial exogenous insulin in order to produce a more physiological profile. One such approach is to modify the insulin formulation. Fast-acting insulin aspart is a modified formulation of insulin aspart containing niacinamide and L-arginine. It has an earlier onset of action than aspart. In an extensive trial programme, this faster aspart
\end{abstract}

Enhanced Digital Features To view enhanced digital features for this article go to https://doi.org/10.6084/ m9.figshare.9582077.

M. Evans $(\varangle) \cdot$ M. Wilkinson · A. Giannpolou Department of Diabetes and Endocrinology, University Hospital Llandough, Penlan Road, Penarth CF64 4XX, UK

e-mail: Marclyndon1@hotmail.com demonstrated similar $\mathrm{HbA1c}$ reductions to those achieved with aspart but superior postprandial glucose reductions, with no increase in hypoglycaemia. Furthermore, administration of faster aspart up to $20 \mathrm{~min}$ after the start of a meal permitted similar glucose control to aspart given preprandially. These data, taken in totality, illustrate the potential role of faster insulin aspart in clinical practice.

Keywords: Diabetes; Faster insulin aspart; Insulin; Insulin aspart

\section{INTRODUCTION}

Postprandial hyperglycaemia is a typical feature of both type 1 and type 2 diabetes [1]. It is an independent risk factor for many complications, including macrovascular disease, and is also associated with an increased risk of retinopathy, cancer and impaired cognitive function in people with type 2 diabetes [1]. The achievement of optimal glycaemic control involves strategies to manage both fasting and postprandial glucose levels [1]. The goal of prandial insulin therapy is to mimic the physiological profile of normal mealtime insulin secretion and action, which translates into attenuated postprandial glucose excursions, without increasing the risk of hypoglycaemia. A variety of nonpharmacological interventions 
may be used to manage postmeal glucose excursions, and as type 2 diabetes progresses, multiple therapies may be employed to address both fasting and postmeal glucose. Prandial insulin is typically deployed as part of a multiple daily insulin regimen in people with type 1 and type 2 diabetes [2], and in the form of a continuous subcutaneous insulin infusion (CSII). Postprandial glucose levels should be measured $1-2 \mathrm{~h}$ after meal ingestion, with a target of $9.0 \mathrm{mmol} / \mathrm{L}(160 \mathrm{mg} / \mathrm{dL})$ as long as hypoglycaemia is avoided [1].

In the healthy individual, there is rapid hepatic insulin exposure with resulting suppression of glucagon-mediated liver glucose production. In contrast, exogenous insulin is absorbed from the subcutaneous tissue and enters the systemic circulation by the capillaries, thus reaching all tissues at the same concentration and the same time, delaying hepatic insulin exposure [3]. A more rapid absorption and onset of action of exogenous prandial insulin has the potential to suppress glucagonmediated hepatic glucose production, thus contributing to enhanced reductions in postprandial glucose levels [4].

Regular human insulin (RHI) entered clinical practice as long ago as 1982; however, while it is structurally identical to endogenously produced insulin, it has a delayed and protracted time-action profile when injected subcutaneously, resulting in the potential for exaggerated early postmeal hyperglycaemia as well as late postprandial hypoglycaemia [5].

These limitations prompted the development, through recombinant engineering of the insulin amino acid sequence, of rapid-acting insulin analogues with a more physiological time-action profile than RHI [5]. Such insulin preparations, including insulin aspart, insulin lispro and insulin glulisine, entered clinical practice in the 1990s, with extensive clinical trial data indicating that these insulins were associated with a reduction in early postprandial hyperglycaemia and late postprandial hypoglycaemia compared with RHI [5].

Despite such innovations, there is still significant room for improvement in the area of prandial insulin pharmacology in terms of the development of an insulin analogue that more closely replicates the glucokinetic and glucodynamic properties of endogenously secreted insulin. This has prompted considerable research efforts that have resulted in the development and introduction into clinical practice of the first new prandial insulin analogue for over 20 years. Faster insulin aspart (FAsp) is now widely available for use in people with both type 1 and 2 diabetes as part of a multiple daily injection regimen and also as part of insulin pump therapy [6]. This article is based on previously conducted studies and does not contain any studies with human participants or animals performed by any of the authors.

\section{THE CLINICAL PHARMACOLOGY OF FASTER ASPART}

FAsp has been specifically developed with a view to deliver its glucodynamic and kinetic effects more quickly compared with current prandial analogue insulins, and thus to more rapidly mimic endogenously produced insulin (Fig. 1). This has been achieved through the inclusion of niacinamide as an absorption modifier and L-arginine as a stabiliser for the insulin aspart (IAsp) preparation [6, 7] (Fig. 2). In a pooled analysis of six euglycaemic clamp studies in people with type 1 diabetes, faster aspart showed an approximate 5-min earlier onset of appearance, early insulin exposure that was twice as high, and a $74 \%$ greater early glucose-lowering effect compared with IAsp [8]. Furthermore, loss of glucose-lowering effect and insulin occurred up to 14 min sooner with FAsp than with IAsp [8]. Similar pharmacological properties have been observed in a Japanese population, the elderly, children and adolescents [9-11].

Since FAsp is essentially IAsp with modified absorption kinetics, it has the same immunogenicity and teratogenicity safety profile as that of IAsp [6].

In elderly subjects, total insulin exposure and the peak insulin concentration of FAsp were $30 \%$ greater than in younger subjects [10]. No age-related differences in glucodynamic effects have been noted, however. Based on these pharmacological properties, FAsp may 


\section{Ultra-rapid insulin: approaching a physiological insulin profile even further}

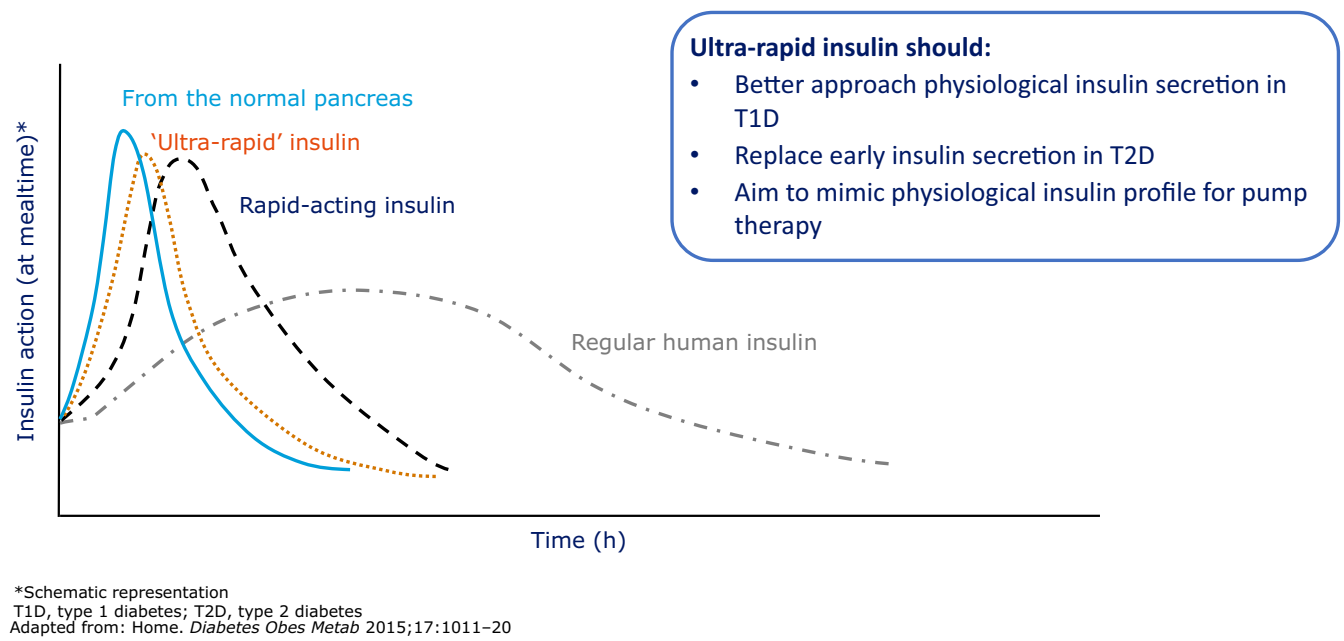

Fig. 1 Approaches used to replicate insulin physiology with a more rapidly acting insulin analogue

Changing the formulation: fast acting aspart is insulin aspart in a new formulation
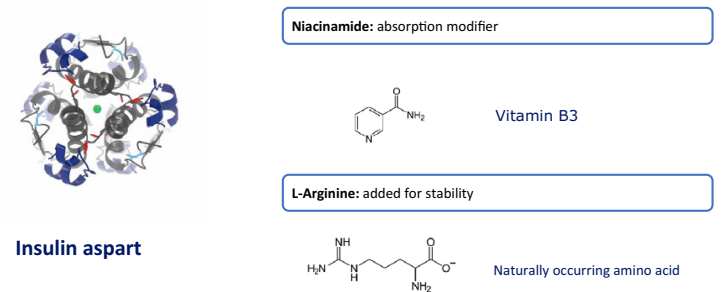

Fig. 2 Formulation of faster insulin aspart

have the potential for both pre- and postprandial dosing and could also be used to address the issues of late postprandial hypoglycaemia and early postprandial hyperglycaemia that may occur with prandial insulin therapy, particularly in the area of the increasingly widespread use of flash glucose monitoring, where such issues may be more readily identified.

When transferring to FAsp from another prandial insulin, close glucose monitoring is recommended, with conversion being made on a unit-for-unit basis [6]. FAsp can be used in pregnancy and during breastfeeding [6]. Since FAsp can be administered up to 20 min before the start of a meal or up to 20 min after starting a meal, the time to onset of action must be considered when prescribing to patients with concomitant conditions where a delayed absorption of food might be expected, such as those with established gastroparesis [6].

\section{FASTER ASPART IN TYPE 1 DIABETES}

The efficacy and safety of FAsp has been studied as part of a multi daily dosing insulin regimen in combination with insulin detemir in adults with type 1 diabetes [12]. Despite this being a noninferiority treat-to-target study, FAsp, dosed at mealtime, yielded a slightly $(0.15 \%)$ greater reduction in HbA1c compared to IAsp after 6 months, which was maintained after 1 year [13]. This advantage in terms of HbA1c seemed to be related to reduced postprandial glucose excursions compared with IAsp. FAsp dosed $20 \mathrm{~min}$ after the start of a meal (postmeal) showed no difference in effect on HbA1c from that of mealtime IAsp [12].

These results were supported by those of a 26-week trial that evaluated FAsp as part of a basal-bolus regimen with insulin degludec [14]. In this study, both mealtime and postmeal FAsp dosing were noninferior to mealtime IAsp in terms of HbA1c. However, there was a significant reduction in the early PPG increments during a standardized meal test with FAsp vs. IAsp. Furthermore, a significantly higher proportion of the subjects $(27.8 \%$ vs. $21.6 \%)$ 
achieved a 1-h PPG target of $<7.8 \mathrm{mmol} / \mathrm{l}$ based on self-measured blood glucose with FAsp compared with IAsp [14]. Both of these studies demonstrated no increase in the overall rate of hypoglycaemia with FAsp compared with IAsp, while a pooled analysis of both studies indicated a $16 \%$ reduction in nocturnal hypoglycaemia with FAsp compared to IAsp [15].

There were, however, some interesting qualitative differences in the distribution of hypoglycaemia between FAsp and IAsp. Although the rates of hypoglycaemia observed during the first hour after a meal were very low, there was an increase with mealtime FAsp vs. IAsp [12, 13]. Conversely, there was a $28 \%$ reduction in hypoglycaemia in favour of mealtime FAsp compared with IAsp 3-4 hours after a main meal [13]. There were no body weight differences between patients on FAsp and IAsp, and no difference in insulin dose was seen $[12,13]$. These data illustrate the potential of FAsp to address the issue of late postprandial hypoglycaemia, while consideration and advice should be given to patients with T1D when initiating FAsp in relation to early postprandial hypoglycaemia.

In children and adolescents with type 1 diabetes [16], FAsp yielded a similar profile to that seen in adults with type 1 diabetes $[12,13]$. Change from baseline in 1-h postprandial glucose increment significantly favoured mealtime FAsp versus IAsp over all meals $(P<0.01$ for all), while there were no significant differences in the overall rate of severe or blood-glucose-confirmed hypoglycaemia, or in insulin dose between FAsp and IAsp, irrespective of dose time or meal [16]. Thus, in adults, children and adolescents with type 1 diabetes, FAsp appears to be a safe prandial insulin, with potential advantages in terms of reduced early postprandial hyperglycaemia and reduced late postprandial hypoglycaemia. Questions, however, remain regarding the effects of exercise on the profile of FAsp in people with type 1 diabetes. For postprandial exercise, anticipation with meal insulin bolus reduction is the recommended method to reduce exercise-associated hypoglycaemia. An ongoing study will compare the effects of IAsp and FAsp on glucose reduction during exercise performed $60 \mathrm{~min}$ or
120 min after breakfast with insulin dosing performed $5 \mathrm{~min}$ before breakfast, and should provide insights into the potential role of FAsp with respect to exercise for people with type 1 diabetes [17].

\section{FASTER ASPART IN INSULIN PUMPS}

FAsp has been studied in the setting of insulin pump therapy [18], with an approximately threefold greater insulin exposure and an approximately $100 \%$ greater glucose-lowering effect in the first 30 min with FAsp compared to IAsp. In a crossover trial in adults with type 1 diabetes, FAsp delivered via an insulin pump produced a greater glucose-lowering effect than IAsp in the $2 \mathrm{~h}$ following a liquid meal [19].

In a 16-week trial, insulin pump therapy with FAsp resulted in similar changes in HbA1c to those achieved with IAsp [20]. FAsp was, however, superior to IAsp in change from baseline in 1-h postprandial glucose increment after a meal test, with statistically significant reductions also observed at $30 \mathrm{~min}$ and $2 \mathrm{~h}$. Furthermore, 1-h postprandial increments were lower with FAsp across all meals, as reflected by an interstitial glucose difference of $0.21 \mathrm{mmol} / \mathrm{l}$.

There was no statistically significant difference in the overall rate of severe or blood-glucose-confirmed hypoglycaemia. There was, however, a numerical imbalance between the number of severe hypoglycaemic episodes suffered by patients on FAsp and the number suffered by patients on IAsp in both the 16-week treatment ( 21 vs 7 ) and the 4 -week run-in period (4 vs 0 ) of the study. As such, when converting IAsp to FAsp in the setting of insulin pump therapy, the faster onset of action of FAsp may translate into a need to reduce the insulin dose, while the reduced postprandial glucose increments and early insulin exposure suggest that FAsp may be particularly useful in closed loop delivery systems. Indeed, FAsp is currently being evaluated in the setting of a closed loop system in young adults with type 1 diabetes [21], and an ongoing study is also assessing the role of FAsp in a dual hormone closed loop system [22]. 


\section{FASTER INSULIN ASPART IN TYPE 2 DIABETES}

The role of FAsp has been studied as part of a basal-bolus insulin regimen in people with type 2 diabetes [23, 24]. In one study [24], the addition of FAsp to insulin degludec, based on a patient-driven titration algorithm, resulted in a greater HbA1c reduction (0.94\%) and larger 2-h PPG increments across all meals (estimated treatment difference $2.48 \mathrm{mmol} / \mathrm{l}$ ) compared to insulin degludec dose titration combined with metformin. As expected with insulin regimen intensification, the hypoglycaemia rate was higher in individuals receiving FAsp (12.8 vs. 2.0 episodes/PYE). Despite this, $43 \%$ of the FAsp/insulin degludec group achieved a HbA1c of $6.5 \%$ without experiencing severe hypoglycaemia, compared with only $6.5 \%$ in the degludec/metformin group.

In another study, FAsp was compared with IAsp in people inadequately controlled with a combination of insulin glargine and oral agents over 26 weeks. Overall glycaemic control improved to a similar extent $(-1.38 \%$ and $-1.36 \%)$ following intensification with either FAsp or IAsp. Significantly greater reductions in the 1-h postprandial glucose increment were observed during a meal tolerance test with FAsp, with an estimated treatment difference of $0.59 \mathrm{mmol} / \mathrm{l}$. Overall hypoglycaemia rates were similar in both groups, although hypoglycaemia rates were slightly higher (2.3 vs 1.5 episodes per patient year) with FAsp during the 0-2 $\mathrm{h}$ postprandial period.

These data support the observations derived from studies of FAsp as part of basal-bolus insulin therapy in people with type 1 diabetes [12-14] - that early postprandial glycaemic excursions were attenuated. While there is no overall excess of hypoglycaemia with FAsp compared with IAsp, there is a slight excess of early postprandial hypoglycaemia with FAsp. Thus, when initating FAsp or switching people over to FAsp from alternative prandial insulins, advice regarding the potential for early postprandial hypoglycaemia needs to be provided.

\section{THE ROLE OF FASTER ASPART IN CLINICAL PRACTICE}

The pharmacological profile of and clinical trial data relating to FAsp $[6,7,12-14,19-24]$ illustrate that this new mealtime insulin formulation has the potential to address significant unmet needs with respect to insulin therapy in people with both type 1 and type 2 diabetes, particularly with respect to early postprandial glucose excursions. This attribute of FAsp in relation to its clinical utility is supported by a recent survey of people with both type 1 and type 2 diabetes managed with multiple daily insulin regimens [25], which found that twothirds of the respondents had-for a variety of reasons-experienced postprandial hyperglycaemia in the week prior to the survey.

The adjustment of the mealtime insulin dose on the basis of carbohydrate meal content and blood glucose monitoring is an important feature of optimal diabetes management. Even with correct dosing, postprandial hyperglycaemia and late postprandial hypoglycaemia are often issues when the duration of insulin action does not appropriately reflect glucose absorption, with the risk of hypoglycaemia being further amplified by physical exertion.

Some people with type 1 diabetes, in an attempt to address postprandial hyperglycaemia, administer repeated "corrective" injections, which can lead to overcompensation and the accumulation of circulating insulin, resulting in an increased risk of hypoglycaemia [26]. The time-action profile for and the clinical trial data on FAsp $[6,7,12-14]$ suggest that this new mealtime insulin may reduce the need for such corrective dosing, translating into both improved glucose control and a reduced burden of hypoglycaemia.

The timing of rapid-acting insulin injections is also a key factor in postmeal glucose control in both type 1 and type 2 diabetes. The typical viewpoint is that postprandial hyperglycaemia occurs due to suboptimal insulin dosing, but it may also occur because the action of the insulin is too slow [27].

The delayed onset of action of exogenously delivered insulin compared with endogenously 
secreted insulin necessitates an injection-tomeal interval to control early postprandial hyperglycaemia. IAsp, insulin glulisine and insulin lispro need to be administered approximately 15-20 min before the start of a meal to best meet postprandial insulin needs [5]. However, in daily life, patients struggle to achieve this, and many use only very short premeal intervals or none at all, or they may even inject insulin immediately after a meal. Indeed, patient source data imply that $24 \%$ of people with type 2 diabetes fail to adhere to insulin dosing timing guidance [28].

Inappropriate meal time insulin dosing can result in an elevated insulin dose requirement [28]. The data used to inform these observations are derived from retrospective surveys, and as such are likely to understate the true extent of the problem.

The more rapid onset of action of and earlier clearance of FAsp $[6,7]$ along with the potential for dosing immediately before or after a meal without incurring detrimental effects in terms of either safety or efficacy [6] may represent a significant therapeutic advance with respect to the challenge of improving prandial insulin dose timing and glucose control. Thus, FAsp appears to have significant potential to improve outcomes in terms of hyperglycaemia, hypoglycaemia and overall glucose control in people with type 1 and type 2 diabetes using multiple daily insulin regimens, which should be formally assessed with data derived from realworld clinical practice.

Insulin pump therapy can achieve improved glycaemic control and reduce the hypoglycaemia risk in people with both type 1 and type 2 diabetes [29, 30]. Insulin pumps aim to deliver insulin in a more physiological manner, with a basal infusion rate supplemented with patientactivated boluses at mealtimes. In addition to the need for better delivery and glucose-monitoring technologies, the utility of insulin pumps is limited by the time-action profiles of current rapid-acting insulin analogues, since there is a lag time between adjustment of infusion and the glucose-lowering effect. Furthermore, the pharmacology of currently used rapid-acting insulin is also a limitation in the development of closed-loop automated insulin delivery systems. In addition, glucose control algorithms have been limited by the time-action profile of rapid-acting insulin for controlling postprandial glucose levels due to the risk for late hypoglycaemia. The use of glucagon in dual hormone systems (glucagon and insulin) may mitigate the risk for hypoglycaemia and improve glucose control [31].

A prandial insulin with a faster onset of action (and a faster offset) has the potential to improve glucose control in people using pumps, and might attenuate the need for dual hormone infusion, with its associated complexity and expense-concepts which should be further evaluated in the real world.

\section{CONCLUSIONS}

FAsp represents a significant evolution of the therapeutic landscape of rapid-acting insulin analogues, as it has the potential to address many of the challenges associated with prandial insulin use in routine clinical practice-particularly those relating to dose timing, postprandial hyperglycaemia and late postprandial hypoglycaemia. Real world data will aid in defining the true clinical value of FAsp.

\section{ACKNOWLEDGEMENTS}

Funding. No funding or sponsorship was received for this study or publication of this article.

Authorship. All named authors meet the International Committee of Medical Journal Editors (ICMJE) criteria for authorship for this article, take responsibility for the integrity of the work as a whole, and have given their approval for this version to be published.

Disclosures. Marc Evans, Mathew Wilkinson and Angeliki Giannpolou have nothing to disclose.

Compliance with Ethics Guidelines. This article is based on previously conducted studies 
and does not contain any studies with human participants or animals performed by any of the authors.

Open Access. This article is distributed under the terms of the Creative Commons Attribution-NonCommercial 4.0 International License (http://creativecommons.org/licenses/ by-nc/4.0/), which permits any noncommercial use, distribution, and reproduction in any medium, provided you give appropriate credit to the original author(s) and the source, provide a link to the Creative Commons license, and indicate if changes were made.

\section{REFERENCES}

1. IDF (2011) Guideline for management of postmeal glucose in diabetes. http://www.idf.org. Accessed March 2019.

2. Davies MJ, D'Alessio DA, Fradkin J, Kernan WN, Mathieu C, Mingrone G7, Rossing P, Tsapas A, Wexler DJ, Buse JB. Management of hyperglycemia in type 2 diabetes, 2018. A consensus report by the American Diabetes Association (ADA) and the European Association for the Study of Diabetes (EASD). Diabetes Care. 2018;41(12):2669-701. https://doi.org/10.2337/dci18-0033.

3. Hermansen K, Bohl M, Schioldan AG. Insulin aspart in the management of diabetes mellitus: 15 years of clinical experience. Drugs. 2016;76(1):41-74.

4. Woerle HJ, Szoke E, Meyer C, Dostou JM, Wittlin SD, Gosmanov NR, Welle SL, Gerich JE. Mechanisms for abnormal postprandial glucose metabolism in type 2 diabetes. Am J Physiol Endocrinol Metab. 2006;290(1):E67-77.

5. Home PD. The pharmacokinetics and pharmacodynamics of rapid-acting insulin analogues and their clinical consequences. Diabetes Obes Metab. $2012 ; 14: 780-8$.

6. European Medicines Agency. Summary of the European public assessment report on Fiasp. Amsterdam: EMA; 2017. https://www.ema.europa.eu/ en/medicines/human/EPAR/fiasp. Accessed 22 July 2019.

7. Buckley ST, Kildegaard J, Hoiberg-Nielsen R, Bekker Jeppesen C, Birk Olsen H, Refsgaard HHF, Ludvigsen S, Ribel U, Hostrup S, Sturis J. Mechanistic analysis into the mode(s) of action of niacinamide in faster-acting insulin aspart. Diabetes Technol Ther. 2016;18(Suppl. 1):A116.

8. Heise T, Pieber TR, Danne T, Erichsen L, Haahr H. A pooled analysis of clinical pharmacology trials investigating the pharmacokinetic and pharmacodynamic characteristics of fast-acting insulin aspart in adults with type 1 diabetes. Clin Pharmacokinet. 2017;56:551-9.

9. Fath M, Danne T, Biester T, Erichsen L, Kordonouri $\mathrm{O}$, Haahr H. Faster-acting insulin aspart provides faster onset and greater early exposure vs insulin aspart in children and adolescents with type 1 diabetes mellitus. Pediatr Diabetes. 2017;18:903-10.

10. Shiramoto M, Nishida T, Hansen AK, Haahr H. Fastacting insulin aspart in Japanese patients with type 1 diabetes: Faster onset, higher early exposure and greater early glucose-lowering effect relative to insulin aspart. J Diabetes Investig. 2018;9:303-10.

11. Heise T, Hovelmann U, Zijlstra E, Stender-Petersen $\mathrm{K}$, Jacobsen JB, Haahr H. A comparison of pharmacokinetic and pharmacodynamic properties between faster-acting insulin aspart and insulin aspart in elderly subjects with type 1 diabetes mellitus. Drugs Aging. 2017;34:29-38.

12. Russell-Jones D, Bode BW, De Block C, et al. Fastacting insulin aspart improves glycemic control in basal-bolus treatment for type 1 diabetes: results of a 26-week multicentre, active-controlled, treat-totarget, randomized, parallel-group trial (onset 1). Diabetes Care. 2017;40:943-50.

13. Mathieu C, Bode BW, Franek E, et al. Efficacy and safety of fast-acting insulin aspart in comparison with insulin aspart in type 1 diabetes (onset 1): a 52-week, randomized, treat-to-target, phase 3 trial. Diabetes Obes Metab. 2018;20:1148-55.

14. Buse JB, Carlson AL, Komatsu M, et al. Fast-acting insulin aspart versus insulin aspart in the setting of insulin degludec-treated type 1 diabetes: efficacy and safety from a randomized double-blind trial. Diabetes Obes Metab. 2018;20:2885-93.

15. De Block C, Carlson AL, Rose L, Gondolf T, GorstRasmussen A, Lane W. Hypoglycemia with mealtime fast-acting insulin aspart vs. insulin aspart across two large type 1 diabetes trials. Diabetes. 2018;67:LB27.

16. Bode B, Iotova V, Kovarenko M, Laffel LM, Rao PV, Deenadayalan S, Ekelund M, Larsen SF, Danne T. Efficacy and safety of fast-acting insulin aspart compared with insulin aspart, both in combination with insulin degludec, in children and adolescents with type 1 diabetes: the onset 7 trial. Diabetes Care. 2019;42(7):1255-62. https://doi.org/10.2337/ dc19-0009. 
17. ClinicalTrials.gov. Comparison of FiAsp and Aspart During Postprandial Exercise in Adults with Type 1 Diabetes. ClinicalTrials.gov identifier: NCT03659799. https://clinicaltrials.gov/ct2/show/NCT03659799. Accessed Aug 2019.

18. Zijlstra E, Demissie M, Graungaard T, Heise T, Nosek L, Bode B. Investigation of pump compatibility of fast-acting insulin aspart in subjects with type 1 diabetes. J Diabet Sci Technol. 2018;12:145-51.

19. Bode BW, Johnson JA, Hyveled L, Tamer SC, Demissie M. Improved postprandial glycemic control with faster-acting insulin aspart in patients with type 1 diabetes using continuous subcutaneous insulin infusion. Diabetes Technol Ther. 2017;19:25-33.

20. Klonoff D, Evans ML, Lane W, et al. A randomized, multicentre trial evaluating the efficacy and safety of fast-acting insulin aspart in continuous subcutaneous insulin infusion in adults with type 1 diabetes (onset 5). Diabetes Obes Metab. 2018. https:// doi.org/10.1111/dom.13610.

21. ClinicalTrials.gov. Closed-Loop with Faster Aspart in Young Adults with Type 1 Diabetes. ClinicalTrials.gov identifier: NCT03212950. https://clinical trials.gov/ct2/show/NCT03212950. Accessed Aug 2019.

22. EU Clinical Trials Register. Dual-Hormone ClosedLoop Glucose Control in Type 1 Diabetes. EudraCT number: 2019-001631-31. https://www.clinicaltrials register.eu/ctr-search/trial/2019-001631-31/DK. Accessed Aug 2019.

23. Bowering K, Case C, Harvey J, et al. Faster aspart versus insulin aspart as part of a basal-bolus regimen in inadequately controlled type 2 diabetes: the onset 2 trial. Diabetes Care. 2017;40:951-7.

24. Rodbard HW, Tripathy D, Vidrio Velázquez M, Demissie M, Tamer SC, Piletič M. Adding fast-acting insulin aspart to basal insulin significantly improved glycaemic control in patients with type 2 diabetes: a randomized, 18-week, open-label, phase 3 trial (onset 3). Diabetes Obes Metab. 2017;19(10):1389-96. https://doi.org/10.1111/dom. 12955.

25. Brod M, Nikolajsen A, Weatherall J, Pfeiffer KM. Understanding post-prandial hyperglycemia in patients with type 1 and type 2 diabetes: a webbased survey in Germany, the UK, and USA. Diabetes Ther. 2016;7:335-48.

26. Peters A, Van Name MA, Thorsted BL, Piltoft JS, Tamborlane WV. Postprandial dosing of bolus insulin in patients with type 1 diabetes: a crosssectional study using data from the T1D Exchange Registry. Endocr Pract. 2017;23:1201-9.

27. Heise $\mathrm{T}$, Meneghini LF. Insulin stacking versus therapeutic accumulation: understanding the differences. Endocr Pract. 2014;20:75-83.

28. Schaper NC, Nikolajsen A, Sandberg A, Buchs S, Bogelund M. Timing of insulin injections, adherence, and glycemic control in a multinational sample of people with type 2 diabetes: a cross-sectional analysis. Diabetes Ther. 2017;8:1319-29.

29. Misso ML, Egberts KJ, Page M, O’Connor D, Shaw J. Continuous subcutaneous insulin infusion (CSII) versus multiple insulin injections for type 1 diabetes mellitus. Cochrane Database Syst Rev 2010;CD005103.

30. Pickup JC, Reznik Y, Sutton AJ. Glycemic control during continuous subcutaneous insulin infusion versus multiple daily insulin injections in type 2 diabetes: individual patient data meta-analysis and meta-regression of randomized controlled trials. Diabetes Care. 2017;40:715-22.

31. Peters TM, Haidar A. Dual-hormone artificial pancreas: benefits and limitations compared with single-hormone systems. Diabet Med. 2018;35:450-9. 